\title{
Reducing Source Current Harmonics Due to BALANCED AND UN-BALANCED VOLTAGE VARIATION WITH FUZZY CONTROLLED D-STATCOM
}

\author{
Mr. K. Ravi Sankar ${ }^{1}$, Mr. Dr.V. Kamaraju ${ }^{2}$ and Mr. Dr. R. Srinivasa Rao ${ }^{3}$ \\ ${ }^{1}$ Assoc. Professor, Department of EEE, Coastal Institute of Technology and Management,Vizag, \\ Andhra Pradesh, India. \\ ${ }^{2}$ Professor, Department of EEE, Mahaveer Institute of Science and Technology, \\ Bandlaguda, Hyderabad, Telangana, India \\ ${ }^{3}$ Professor, Department of EEE, University College of Engineering, JNTUK, Kakinada, \\ Andhra Pradesh
}

\begin{abstract}
Electricity demand has been increasing due to the increase of population and industries. Hence it is very important to feed the demand with quality of generation. Improvement of power quality is the greater concern in advanced power system element, it is essential to congregate the need of energy by employ the renewable energy generating sources like $p v$, fuel cell, biomass, wind, etc and utilizing many more applications like grid interconnected systems, power quality improvement. The situations like harmonic, reactive power exchanging, power factor correction, balanced and un-balanced voltage variations \& so on, due to greater effect on highly susceptible loads are to be encouraged in power distribution system. To enhance these circumstances, custom power appliances are used to achieve high grid voltage stability. In this paper, a voltage distortions controlled D-STATCOM based on fuzzy controller is a meticulous power appliance for enhancing harmonic distortions from high power semiconductor switching device, exchanging the both active \& reactive power, defend the gird stability by implementing DG technology, to regulate the power quality issues in power distribution network. This paper implements the comparative analysis of PI and Fuzzy controlled DSTATCOM with energy backup scheme, controlled by pwm control technique and founded that fuzzy based IPQ theory for generation of reference current signals improves the power quality than PI based IPQ theory and this analysis is dynamically evaluated with Matlab/Simulink.
\end{abstract}

\section{KEYWORDS}

D-STATCOM, balanced-un-balanced voltage variations, fuzzy controller and Harmonics.

\section{INTRODUCTION}

Renewable generation from wind and sun has enlarged considerably throughout past few years and forms a significance proportion of the whole generation within the grid. This renewable generation is focused in a very few states, to the extent that it can't be known as marginal generation and high thought must run to balance the variability of such generation. There's a formidable programme for increase of such Renewable Generation and so, it's imperative to figure out how forward for facilitating massive scale integration of such variable Renewable Energy Sources (RES), keeping the protection of the grid. Moreover, as we tend to move towards a tighter frequency band, it becomes even more difficult to balance this variable RES. Generation 
from RE Sources depends on nature, i.e. wind rate and sunshine. The variability of RES power will be self-addressed through improved statement techniques that are still evolving. Once the proportion of RES becomes important, special attention must be paid to accurately forecast their output. The changes in RES might affect the electrical network. There has been excessive traction of non-sinusoidal current and voltage in electrical network, due to excessive usage of non-linear masses. Power quality could also be marked in synchronous and non- synchronized phenomena and this will result into visiting synchrononised with ac wave at power frequency.

A group of controllers along known as Custom Power Devices (CPD), that embody the DSTATCOM (distribution static compensator), The DSTATCOM, a shunt connected device will take care of the quality issues in the current it contains a de electrical condenser. Three phase electrical converter module ac filter ,coupling electrical device and an impression strategy the electronic block of D-STATCOM is the voltage sourced electrical converter, which converters an input dc voltage into three phase output voltage in harmonic. The D-STATCOM puts electrical convert, convert the dc-link voltage $\mathrm{Vdc}$ on electrical condenser. It can be presumed $\mathrm{D}$ STATCOM may be treated as voltage control supply.

The proposed fuzzy based instant power theory is valid for pure sin or non-sinusoidal and balanced or unbalanced power systems and were later planned [4-8].The part of the output voltage of the thyristor-based electrical converter, $\mathrm{Vi}$, is controlled within the same approach because the distribution system voltage, Vs. The proposed DSTATCOM relies on the fuzzy based instant power theory; it provides smart compensation characteristics in steady state furthermore as transient states compared to Proportional Integral (PI) based IPT Controller [9]. The FUZZY based instant power theory generates the reference currents needed to compensate the distorted line current harmonics and reactive power accurately when compared to the PI controller since it reduces both error and change in error and also large variation in errors. Hence the proposed concept has been analyzed with FUZZY controlled IPT and compared its advantages with the PI controlled IPT.

\section{Principal Of The D-Statcom}

The D-STATCOM is that the solid-state-based power device version of the SVC. The construct of the D-STATCOM was planned by Gyugyi in 1976. In operation as a shunt-connected SVC, its electrical phenomenon or inductive output currents is controlled severally from its connected AC bus voltage. Attributable to the fast-switching characteristic of power converters, the DSTATCOM provides a lot of quicker response as compared to the SVC.

The shunt compensator will live the PCC voltages and within reference current generations algorithms with none drawback as these voltages area unit pure sinusoids. This may not be attainable in actual systems where the masses area with at top of the feeder.

The PCC voltage is distorted by the switch frequency harmonics generated by D-STATCOM. There will be switch and resistive loss within the D-STACOM circuit. These losses should be proved by the system. We have a tendency to should so fitly modify the reference current generation rule to accommodate of these factors. Finally, to supply a path for the harmonic current generated by the CMLI primarily based VSI realizing the D-STATCOM to flow; we have a tendency to should place further filters within the circuit.

In addition, within the event of a fast amendment in system voltage, the electrical condenser voltage doesn't amendment instantaneously; thus, the D-STATCOM effectively reacts for the specified responses. As an example, if the system voltage drops for any reason, there's an inclination for the D-STATCOM to inject electrical phenomenon power to support the unfit 
voltages. On paper, the ability device used within the D-STATCOM is either a VSC or a currentsource device (CSC). In observe, however, the VSC is most popular owing to the bidirectional voltage-blocking capability needed by the ability semiconductor devices utilized in CSCs. to realize this type switch characteristic, an extra diode should be connected nonparallel with a standard semiconductor switch, instead the organic structure of the semiconductor should be changed. Each of those alternatives increase the physical phenomenon losses and total system value. In general, a CSC derives its terminal power from a current supply, i.e., a reactor. As compared, a charged reactor is way lousier than a charged electrical condenser. Moreover, the VSC needs a current-source filter at its AC terminals that is of course provided by the coupling electrical device outpouring inductance, whereas extra electrical condenser banks square measure required at the AC terminals of the CSC. Finally, the VSCs will operate with higher potency than the CSCs waste high-energy applications.

A suitable VSC is chosen supported the subsequent considerations: the voltage rating of the facility network, this harmonic demand, the system complexness, etc. Basically, the DSTATCOM system is comprised of 3 main parts: a VSC, a group of coupling reactors or a transformer, and a controller. The inductances of the increased power transformers will act as coupling reactors, while at a very high voltage system. The very purpose of the coupling inductors is to strain these elements, that square measure generated primarily by the output voltage facility. The voltage quality downside may be a concern, wherever the D-STASTCOM is connected to the facility at PCC. Voltages, currents and square measure fed into controller to compare with commands.

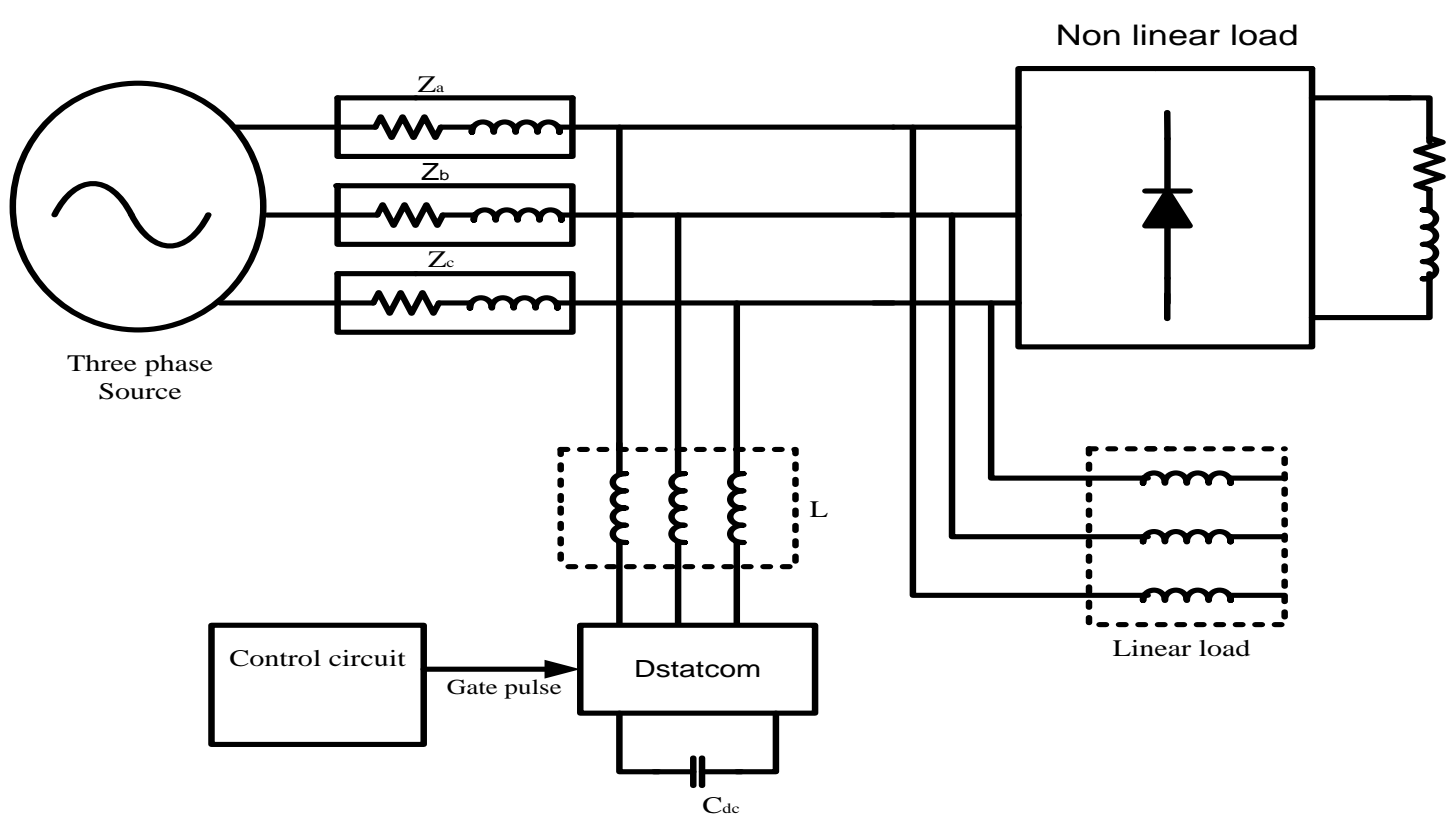

Fig.1.Basic Block Diagram of IPT Controlled DSTATCOM with Reactive and Harmonic Loads

The control performs as feedback control and brings a collection of changes consequently. The diagram illustration of the D-STATCOM system with non-linear load is illustrated in Fig.1. The VSC is described as a perfect supply related to internal loss that connected to the ac power via coupling reactors. The exchange of the real power and reactive power between d-statcom and additional able area unit typically controlled by the amplitude sections of the output voltage. Within the case of a perfect lossless power convertor, the output voltage of the convertor is controlled to be in section thereupon of the ability system. During this case, there's no real power circulated within the D-STATCOM; so, a true power supply isn't required. To work the DSTATCOM in electrical phenomenon mode or volt-ampere generation, $+Q$, the magnitude of the 
convertor output voltage is controlled to be larger than the voltage at the PCC. In distinction, the magnitude of the output voltage of the convertor is controlled to be but that of the ability system at the PCC on order to soak up reactive power or to work the D-STATCOM in inductive mode, Q. However, in observe, the convertor is related to internal losses caused by non-ideal power semiconductor devices and passive elements. As a result, with none correct controls, the condenser voltage are going to be discharged to compensate these losses, and can constantly decrease in magnitude. To regulate the condenser voltage, very low phase shift $\delta$ is introduced between the device voltage and therefore the installation voltage.

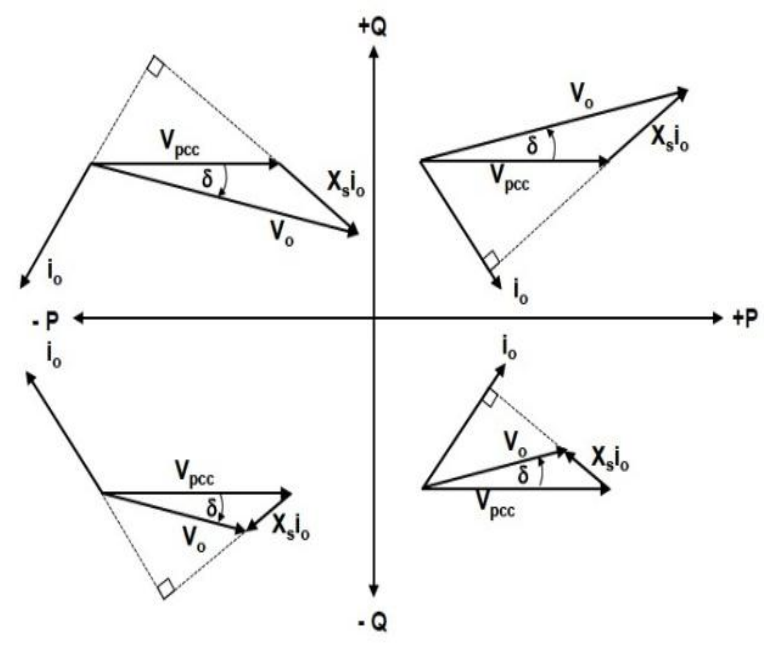

Fig .2. Phasor Diagram for Power Exchanges in Four Quadrants of DSTATCOM

A small lag of the device voltage with relevance the voltage at the PCC causes real power to ensue the ability system to the D-STATCOM, whereas the active power is transferred from the DSTATCOM to the ability system by dominant the device voltage in order that it leads the voltage at the PCC. Fig .2. illustrates phasor diagrams of the voltage at the PCC, device output current and voltage all told four quadrants of the PQ plane.

\section{Control Strategy}

From the very beginning of the electrical distribution system, voltage regulation, reactive power burden and unbalanced loading of the phases are major problems faced by the power engineers. With the advent of custom power devices and FACTS technology, the concept of DSTATCOM has appeared which has served the purpose of dynamic reactive power compensation. With a few modifications, DSATACOM can also be used for neutral current compensation, load balancing and voltage regulation.

For reactive power compensation, DSTATCOM provides reactive power as required by the load and thus the supply current remains at unity power issue (UPF). Since solely real power is being provided by the supply, load equalization is achieved by creating the supply reference current balanced.

Control strategy plays an important role in overall performance of the compensating device. The management of a compensating device is completed in 3 stages. Within the 1st stage, the essential voltage and current signals area unit detected power transformers (PT's), CT's, Hall-effect sensors, and isolation amplifiers to assemble correct system info. Within the second stage, compensating commands in terms of current or voltage levels area unit derived supported completely different management ways and device configurations. Within the third stage of management, the gating signals for the solid-state devices of the compensating devices area unit 
generated either in open loop or closed-loop system. Among the foremost in style schemes area unit PWM, whereas for closed-loop system, unipolar pulse breadth modulation management technique is that the most typical kind of pursuit management for CMLI based mostly shunt compensators.

There are unit several management approaches offered for the generation of reference supply currents for the management of VSC of DSTATCOM for three-phase, three-wire system within the literature viz.

1. Instantaneous Power Theory ( IP Theory),

2. Synchronous Reference Frame (SRF) Theory,

3. Id-Iq Theory,

4. Modified Id-Iq Theory.

5 .

Among the different control techniques applied to three-phase three-wire compensators, the SRF based technique is found to be suitable for different topologies of DSTATCOM. The IRP theory is simple and proven to be one of the best performances under various operating conditions and hence this theory is used for the control of proposed H-bridge DSTATCOM.

\subsection{Proposed Instantaneous Power Theory (IPT)}

IP theory was primarily planned by Akagi. This theory relies on the transformation of 3 section quantities to 2 section quantities in $\alpha-\beta$ frame and also the calculation of instant active and reactive power during this frame. A basic diagram of this theory is shown in fig.3. detected inputs $V s a, V s b, V s c$ and $i L a, i L b, i L c$ are fed to the controller, and these quantities ar processed to come up with reference current commands $\left(i_{S a}^{*}, i_{S b}^{*}, i_{S c}^{*}\right)$, that are fed to a pulse dimension modulation (PWM) signal generator to come up with final shift signals fed to the D-STATCOM; Hence this block control the D-Statcom.

The source voltages can be given as:

$$
\begin{aligned}
& v a=V m \sin (\omega t) \\
& v b=V m \sin (\omega t-2 \pi / 3) \\
& v c=V m \sin (\omega t-4 \pi / 3)
\end{aligned}
$$

Load currents of the system given as:

$$
\begin{aligned}
i_{L a} & =\sum I_{L a n} \sin \left\{n(\omega t)-\theta_{a n}\right\} \\
i_{L b} & =\sum I_{L b n} \sin \left\{n(\omega t-2 \pi / 3)-\theta_{b n}\right\} \\
i_{L c} & =\sum I_{L c n} \sin \left\{n(\omega t-2 \pi / 3)-\theta_{c n}\right\}
\end{aligned}
$$

In 3 phase coordinates, a, b, and c axes are fixed on the same plane, with the phase difference by $2 \pi / 3$. The instantaneous space vectors $v a$ and $i L a$ are set on the " $a$ " axis, and their amplitude varies in positive and negative directions with time. This is true for the other two phases also. By using Park's transformation can be transformed to these phases. 

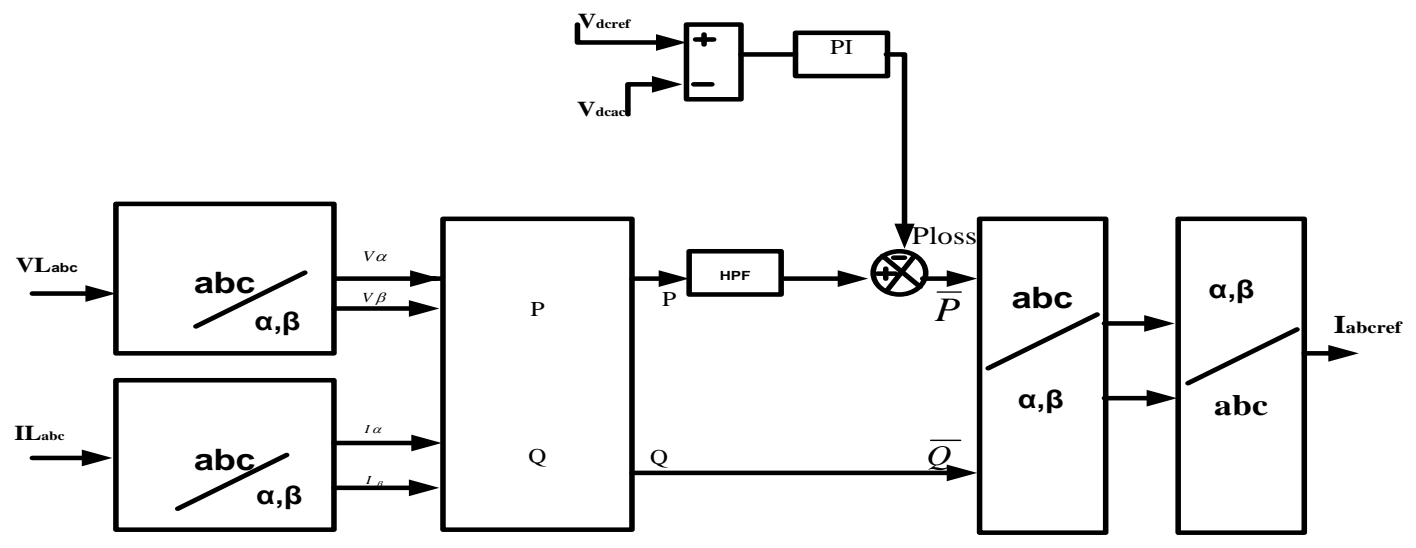

Fig .3. Block Diagram of Proposed Control Strategy- Instantaneous Power Theory

$$
\begin{aligned}
& {\left[\begin{array}{l}
v_{\alpha} \\
v_{\beta}
\end{array}\right]=\sqrt{\frac{2}{3}}\left[\begin{array}{ccc}
1 & -1 / 2 & -1 / 2 \\
0 & \sqrt{3} / 2 & -\sqrt{3} / 2
\end{array}\right]\left[\begin{array}{l}
v_{a} \\
v_{b} \\
v_{c}
\end{array}\right]} \\
& {\left[\begin{array}{l}
i_{\alpha} \\
i_{\beta}
\end{array}\right]=\sqrt{\frac{2}{3}}\left[\begin{array}{ccc}
1 & -1 / 2 & -1 / 2 \\
0 & \sqrt{3} / 2 & -\sqrt{3} / 2
\end{array}\right]\left[\begin{array}{l}
i_{a} \\
i_{b} \\
i_{c}
\end{array}\right]}
\end{aligned}
$$

Where $\alpha$ and $\beta$ axes are the orthogonal coordinates. Conventional instantaneous power for threephase circuit can be defined as

$$
p=v_{\alpha} i_{\alpha}+v_{\beta} v_{\beta}
$$

Where $\mathrm{p}$ is equal to conventional equation

$$
p=v_{a} i_{a}+v_{b} i_{b}+v_{c} i_{c}
$$

Hence the IRP can be given as

$$
q=-v_{\beta} i_{\alpha}+v_{\alpha} i_{\beta}
$$

Therefore, in matrix form, instantaneous real and reactive power are given as

$$
\left[\begin{array}{l}
p \\
q
\end{array}\right]=\left[\begin{array}{cc}
v_{\alpha} & v_{\beta} \\
-v_{\beta} & v_{\alpha}
\end{array}\right]\left[\begin{array}{l}
i_{\alpha} \\
i_{\beta}
\end{array}\right]
$$

The $\alpha-\beta$ currents can be obtained as

$$
\left[\begin{array}{l}
i_{\alpha} \\
i_{\beta}
\end{array}\right]=\frac{1}{\Delta}\left[\begin{array}{cc}
v_{\alpha} & v_{\beta} \\
-v_{\beta} & v_{\alpha}
\end{array}\right]\left[\begin{array}{l}
p \\
q
\end{array}\right]
$$

Where

$$
\Delta=v_{\alpha}^{2}+v_{\beta}^{2}
$$

Instantaneous active and reactive powers $p$ and $q$ can be decomposed into an average (dc) and an oscillatory component.

$$
\begin{aligned}
& p=\bar{p}+\tilde{p} \\
& q=\bar{q}+\tilde{q}
\end{aligned}
$$

Where $\bar{p}$ and $\bar{q}$ are the average (dc) part and $\tilde{p}$ and $\tilde{q}$ are the oscillatory (ac) part of these real and reactive instantaneous powers. Reference source currents are calculated to compensate the 
reactive power and the oscillatory component of the instantaneous active power. Therefore, the reference source currents $i_{s \alpha}^{*}$ and $i_{s \beta}^{*}$ in $\alpha-\beta$ coordinate are expressed as

$$
\left[\begin{array}{l}
i_{s \alpha}^{*} \\
i_{s \beta}^{*}
\end{array}\right]=\frac{1}{\Delta}\left[\begin{array}{cc}
v_{\alpha} & -v_{\beta} \\
v_{\beta} & v_{\alpha}
\end{array}\right]\left[\begin{array}{l}
\bar{p} \\
0
\end{array}\right]
$$

Theses currents can be transformed in a-b-c quantities to find the reference currents in a-b-c coordinates using inverse transformation.

$$
\left[\begin{array}{l}
i_{s a}^{*} \\
i_{s b}^{*} \\
i_{s c}^{*}
\end{array}\right]=\sqrt{\frac{2}{3}}\left[\begin{array}{ccc}
1 / \sqrt{2} & 1 & 0 \\
1 / \sqrt{2} & -1 / 2 & \sqrt{3} / 2 \\
1 / \sqrt{2} & -1 / 2 & -\sqrt{3} / 2
\end{array}\right]\left[\begin{array}{c}
i_{0}^{*} \\
i_{s a}^{*} \\
i_{s \beta}^{*}
\end{array}\right]
$$

Where $i_{0}^{*}$ is the zero sequence components, which is zero in three- phase three wire system. The generated reference currents are compared with actual currents by using Hysteresis current controller for generation of pulses.

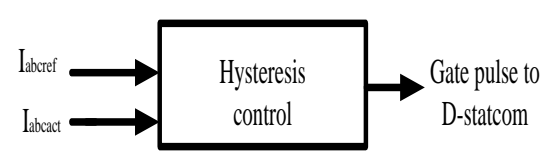

Fig .4. Generation of Gating pulses

\section{FlC CONTROLleR}

The identified power quality and harmonics problems can be effectively reduced by controlling D-STATCOM. Fuzzy logic is wide employed in controlling technique. The word "fuzzy" maintain fact that the logic concerned will wear down ideas that can't be expressed as "true" or "false" however rather as "partially true". Though various approaches like genetic algorithms and ANN will perform even as well as formal logic in several cases, formal logic has the advantage that the answer to the matter is forged in terms that human operators will perceive, so their expertise is employed in the look of the managementler of prognosticative current control. The linguistic variables area unit outlined as (NB, NM, NS, Z, PS, PM, PB) that means negative big, negative medium, negative small, zero, positive small, positive medium and positive big. The membership functions area unit shown in Fig.5.

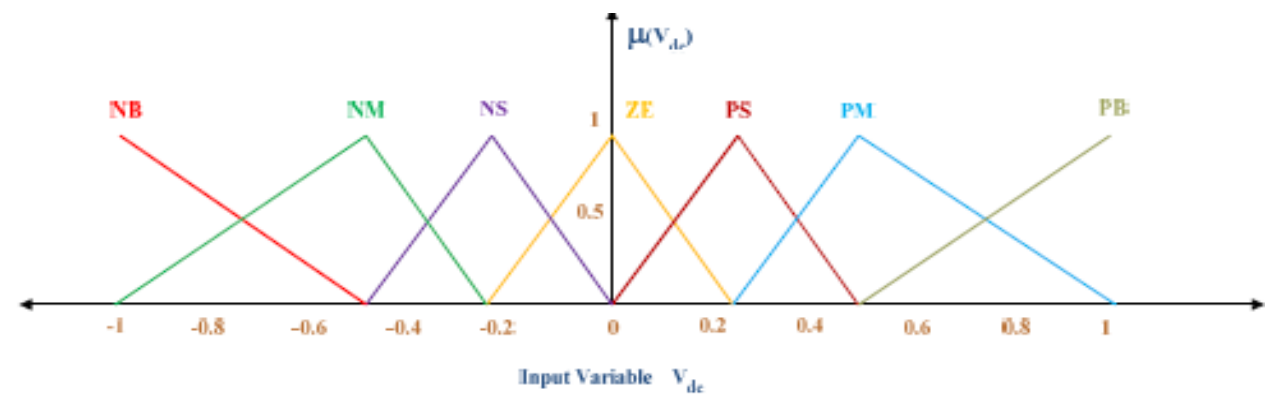

(a) 


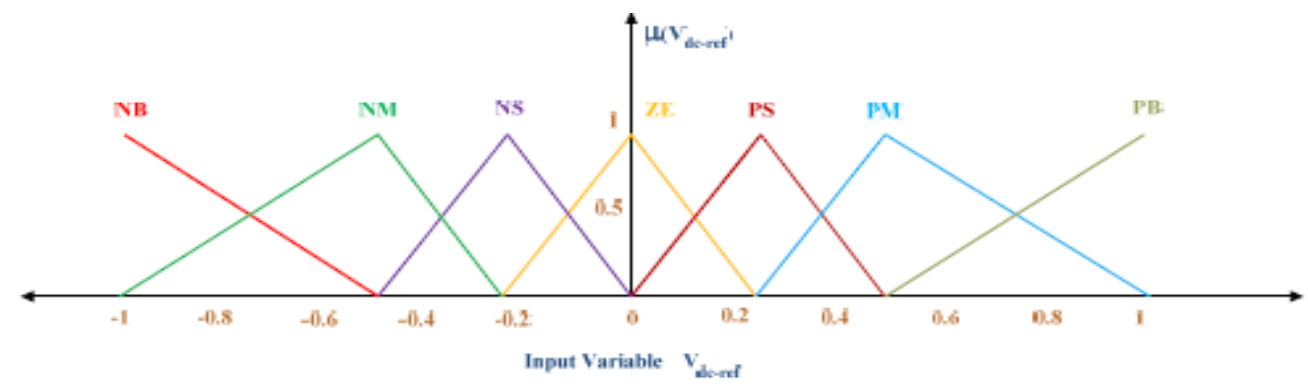

(b)

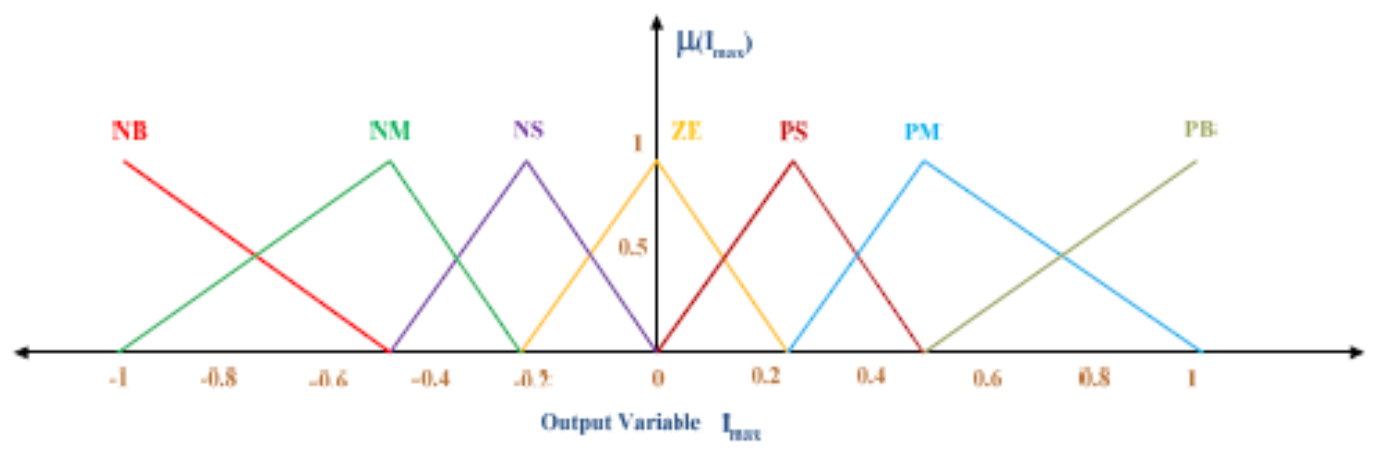

(c)

Fig.5. (a) Input ANF normalized membership function; (b) Input Vdc-ref Normalized Membership Function; (c) Output Imax Normalized Membership Function.

Table 1. The Membership functions for FLC

\begin{tabular}{|c|c|c|c|c|c|c|c|}
\hline E & NB & NM & NS & Z & PS & PM & PB \\
\hline PB & Z & PS & PM & PB & PB & PB & PB \\
\hline PM & NS & Z & PS & PM & PB & PB & PB \\
\hline PS & NM & NS & Z & PS & PM & PB & PB \\
\hline Z & NB & NM & NS & Z & PS & PM & PB \\
\hline NS & NB & NB & NM & NS & Z & PS & PM \\
\hline NM & NB & NB & NM & NM & NS & Z & PS \\
\hline NB & NB & NB & NB & NB & NM & NS & Z \\
\hline
\end{tabular}

From the table I, each error and its change in error is divided into $7 \mathrm{X} 7$ membership functions as:

Negative Big (NB), Negative Medium (NM), Negative Small (NS), Zero (Z), Positive Small (PS), Positive Medium $(P M)$ and Positive Big $(P B)$.

\section{Matlab/Simulink Analysis}

Here simulation is carried out in different cases.

1). Performance of D-Statcom at Balanced Sinusoidal Source Voltage.

2). Performance of D-Statcom at Balanced Non-Sinusoidal Source Voltage.

3). Performance of D-Statcom at Un-Balanced Sinusoidal Source Voltage. 
4). Performance of PI Controlled D-Statcom at Un-Balanced Non-Sinusoidal Source Voltage.

5). Performance of FUZZY Controlled D-Statcom at Un-Balanced Non-Sinusoidal Source Voltage.

\section{Case I: Performance of D-Statcom at Balanced Sinusoidal Source Voltage.}

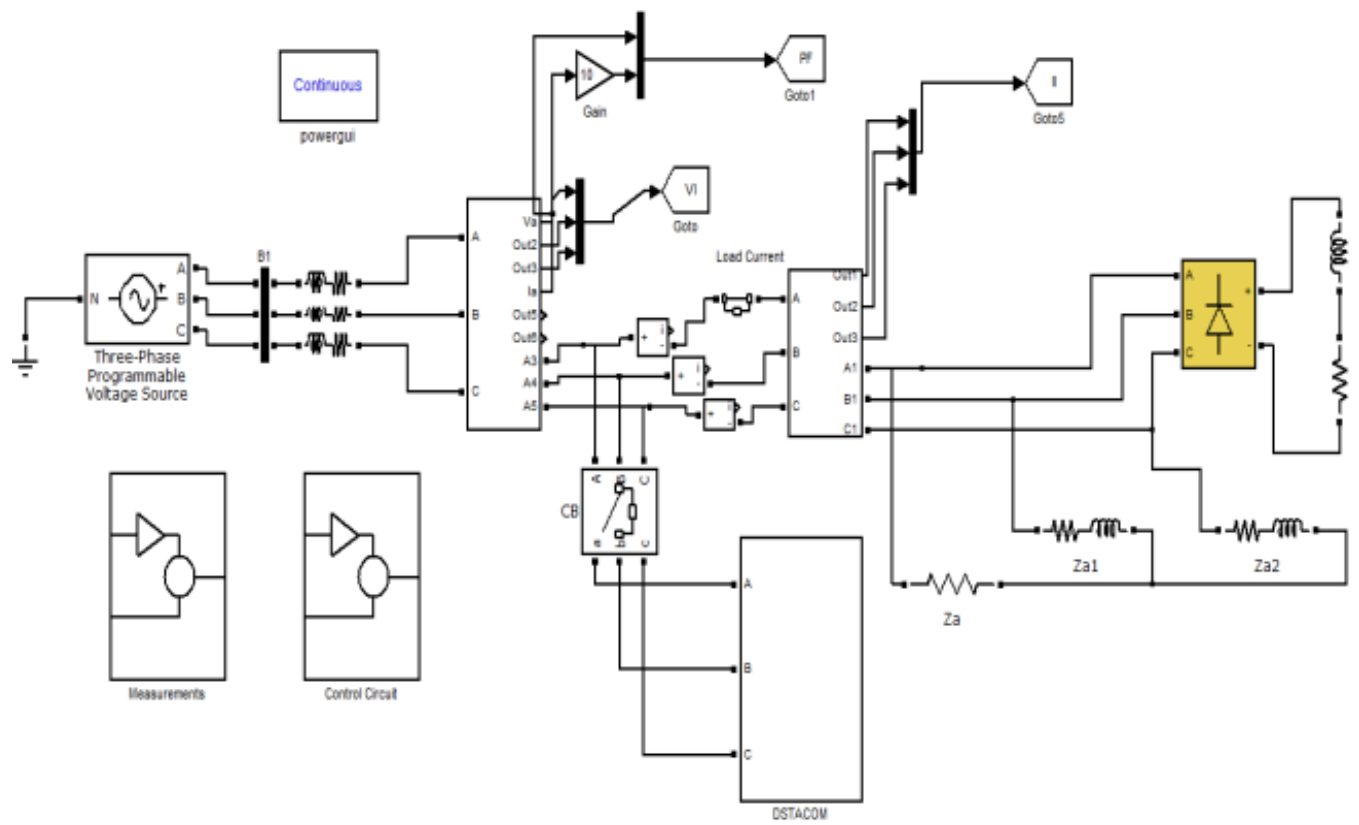

Fig.6. Simulink model of D-Statcom at Balanced Sinusoidal Source Voltage

Fig.6. Shows The Matlab/Simulink Model Of Power System Network With D-Statcom At Balanced Sinusoidal Source Voltage.

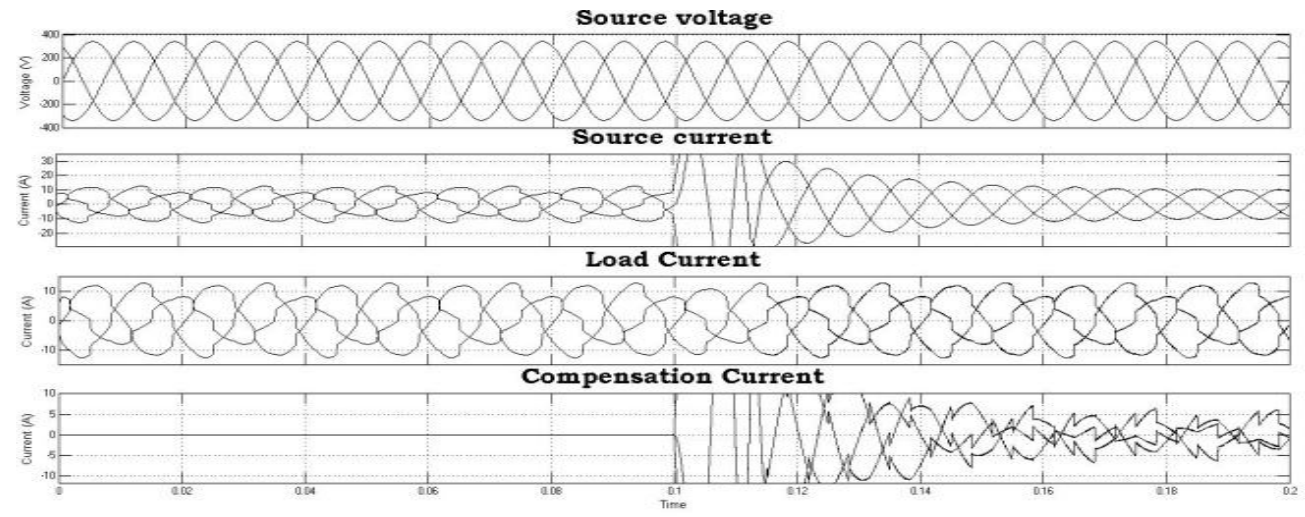

Fig.7. Simulated output wave forms of Source voltage, Source, Load and Compensation Current.

Fig.7. Shows The Simulated Output Wave Forms Of The Source Voltage And Source Current, Load Current And Compensation Current At Balanced Sinusoidal Source Voltage. 


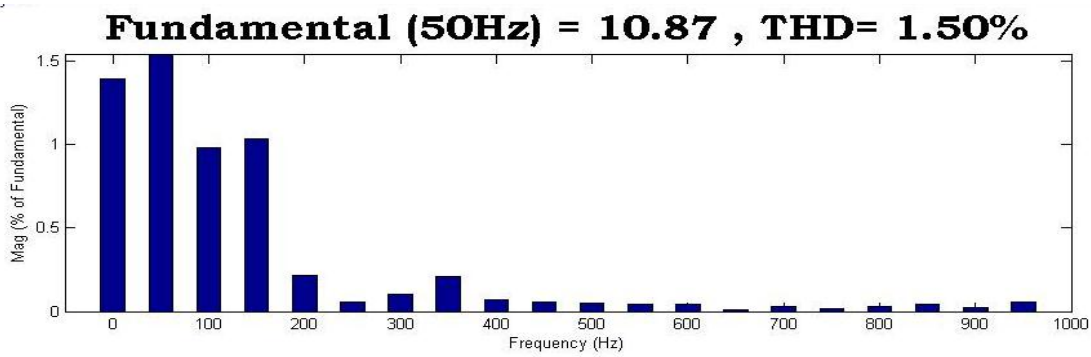

Fig.8. THD of Source Current.

Fig.8.Shows the Total Harmonic Distortion of Source Current at Balanced Sinusoidal Source Voltage.

\section{Case II: Performance of D-Statcom at Balanced Non-Sinusoidal Source Voltage.}

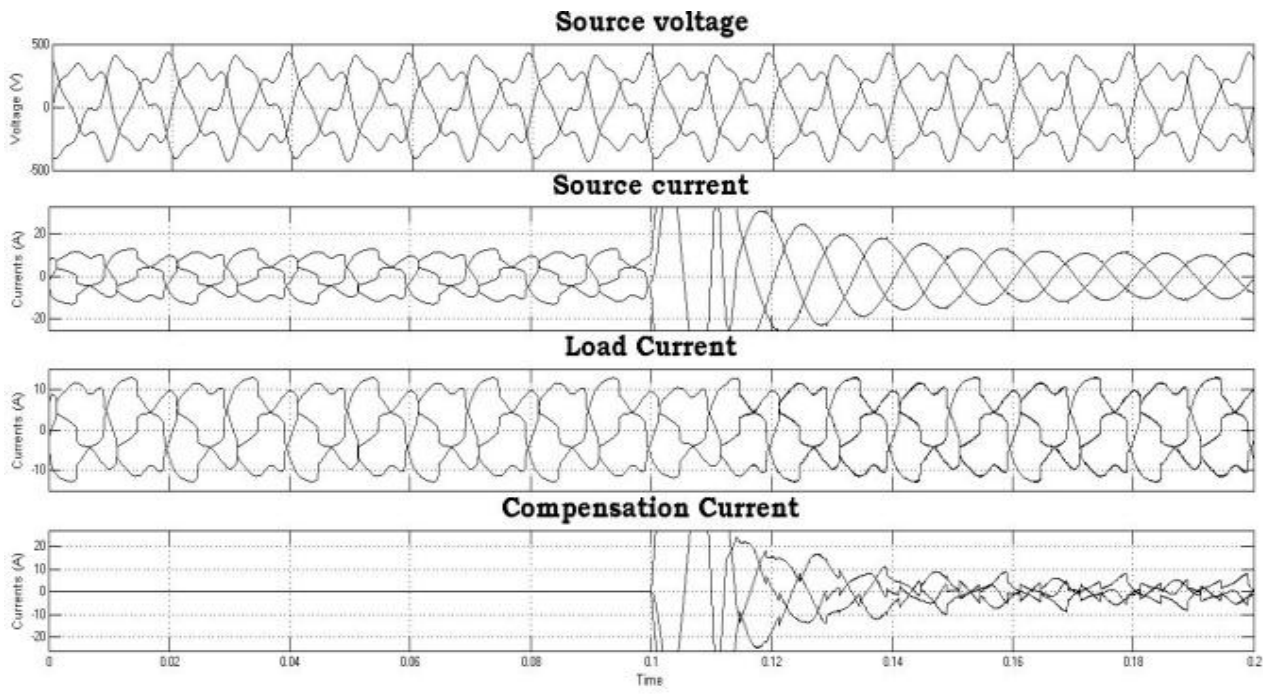

Fig.9. Simulated output wave forms of Source voltage, Source current, Load current and Compensation Current

Fig.9. shows the simulated output wave forms of source voltage and current load current and compensation current at balanced non-sinusoidal source voltage.

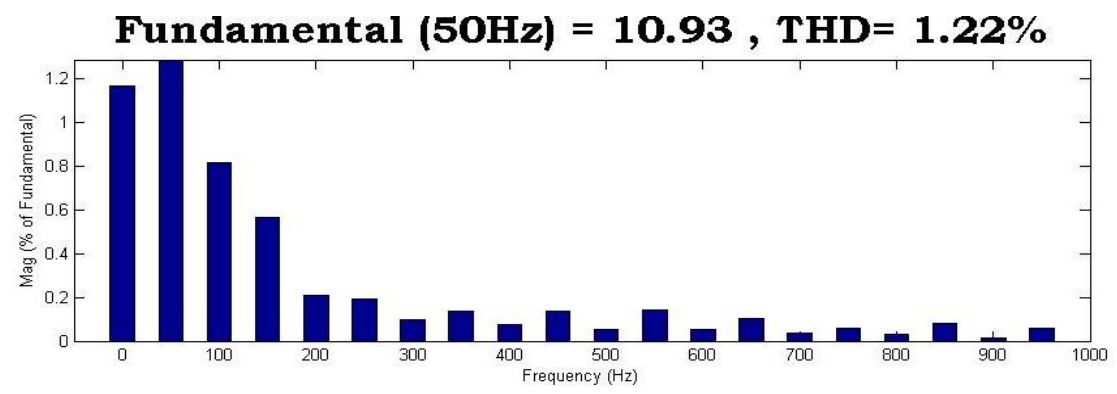

Fig.10. THD of Source Current

Fig.10. shows the total harmonic distortion of source current at balanced non- sinusoidal source voltage. 


\section{Case III: Performance of D-Statcom at Un-Balanced Sinusoidal Source Voltage.}

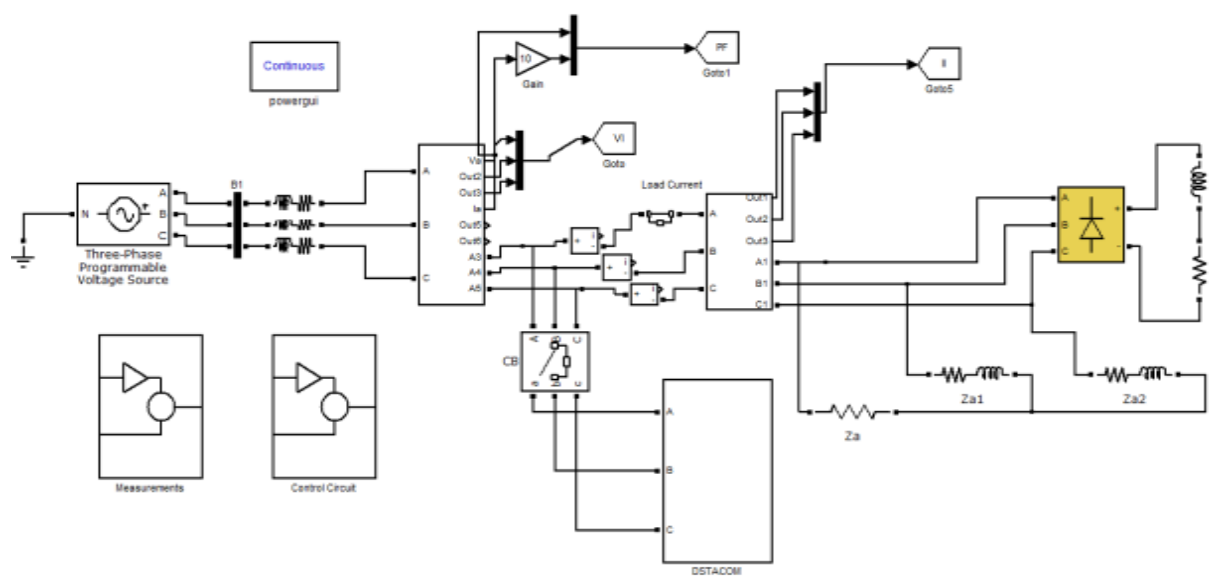

Fig.11. Simulink model of D-Statcom at Un-Balanced Sinusoidal Source Voltage

Fig.11. shows the Matlab/Simulink model of power system network with D-STATCOM at unbalanced sinusoidal source voltage.

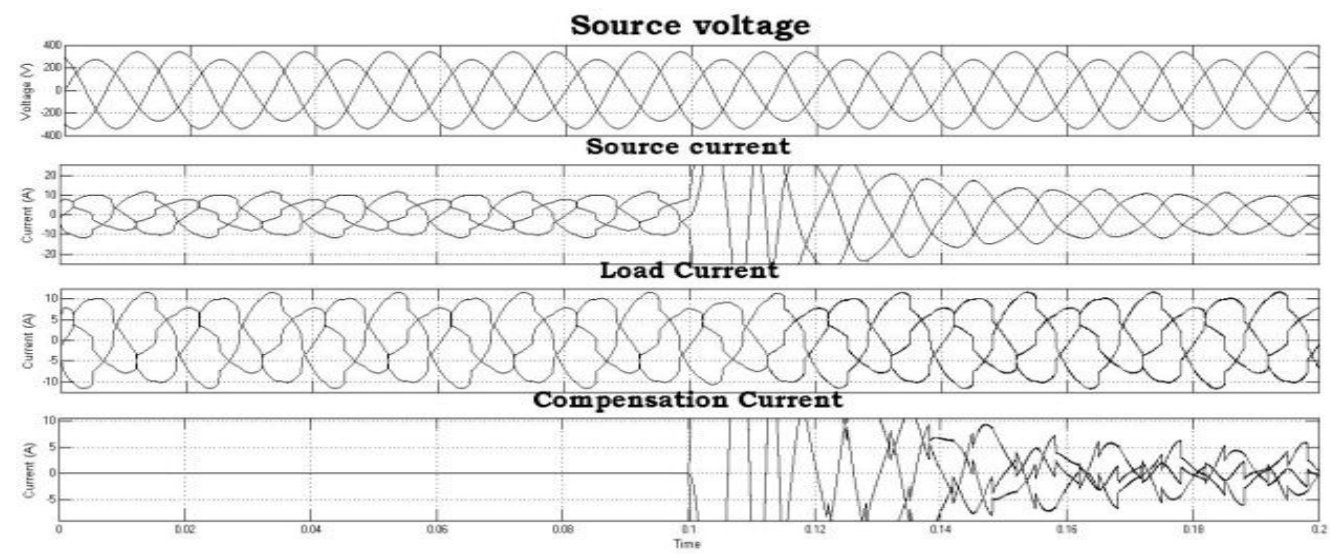

Fig.12. Simulated output wave forms of Source voltage, Source current, Load current and Compensation Current

Fig.12. shows the output wave forms of source voltage and current, load current and compensation current at un-balanced sinusoidal source voltage.

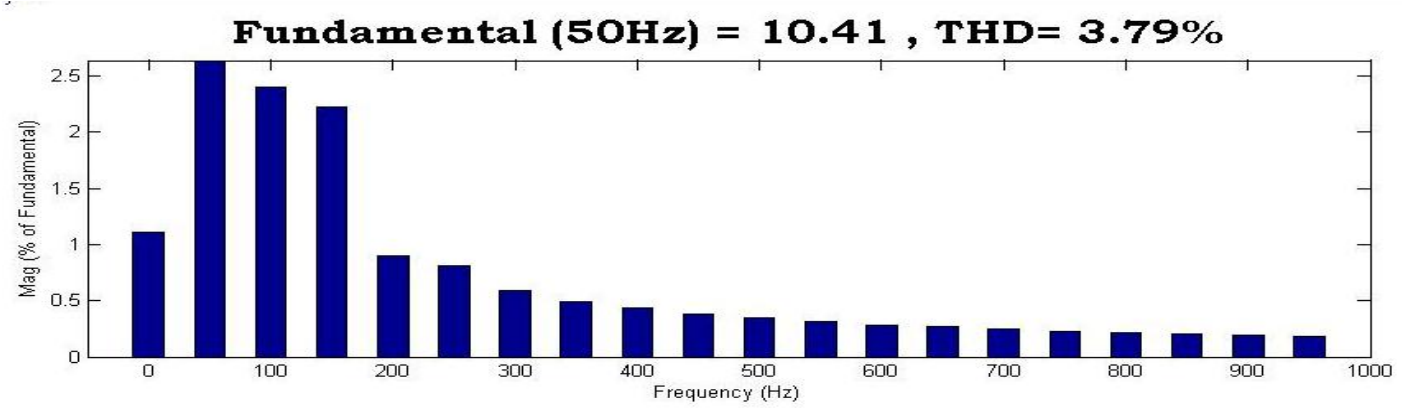

Fig.13.THD of Source Current 
International Journal on Cybernetics \& Informatics (IJCI) Vol. 5, No. 1, February 2016

Fig.13. shows the total harmonic distortion of source current at un-balanced sinusoidal source voltage shows $3.79 \%$.

Case IV: Performance of PI Controlled D-Statcom at Un-Balanced Non-Sinusoidal Source Voltage.
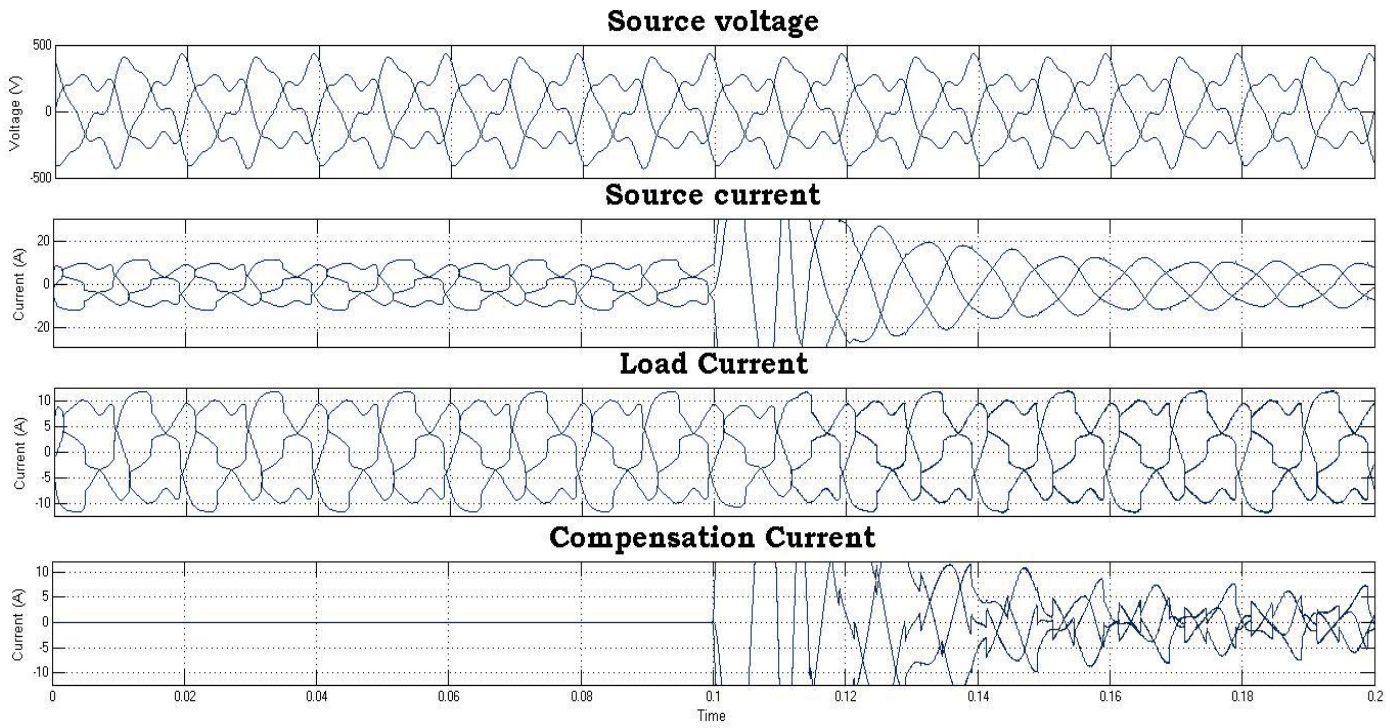

Fig.14. Simulated output wave forms of Source voltage, Source current, Load current and Compensation Current

Fig.14. shows the output wave form of source voltage and current, load current and compensation current at un-balanced non-sinusoidal source voltage with PI Controller.

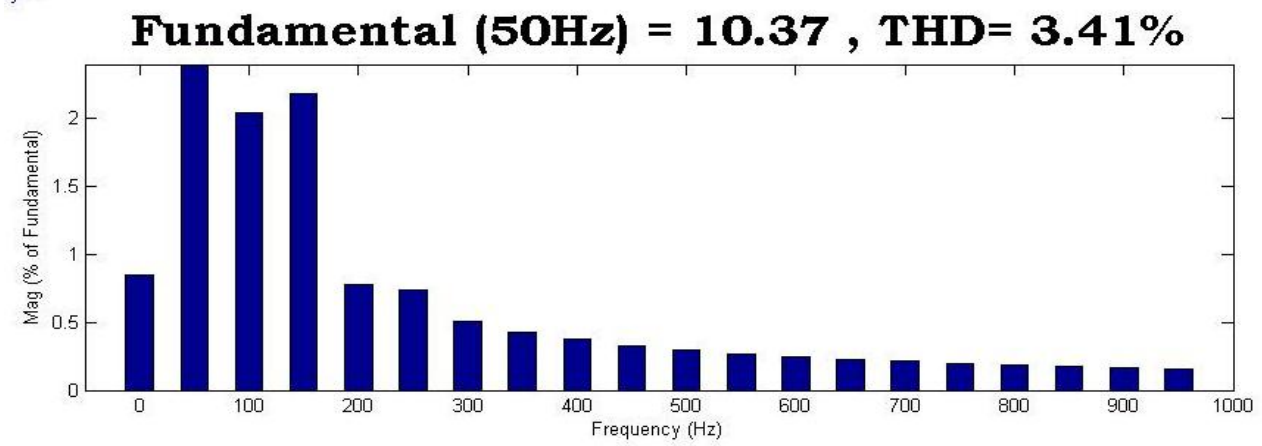

Fig.15. THD of Source Current

Fig.15.shows the total harmonic distortions of source current at un-balanced non-sinusoidal source voltage with PI Controller 
Case V: Performance of FUZZY controlled D-Statcom at Un-Balanced Non-Sinusoidal Source Voltage.

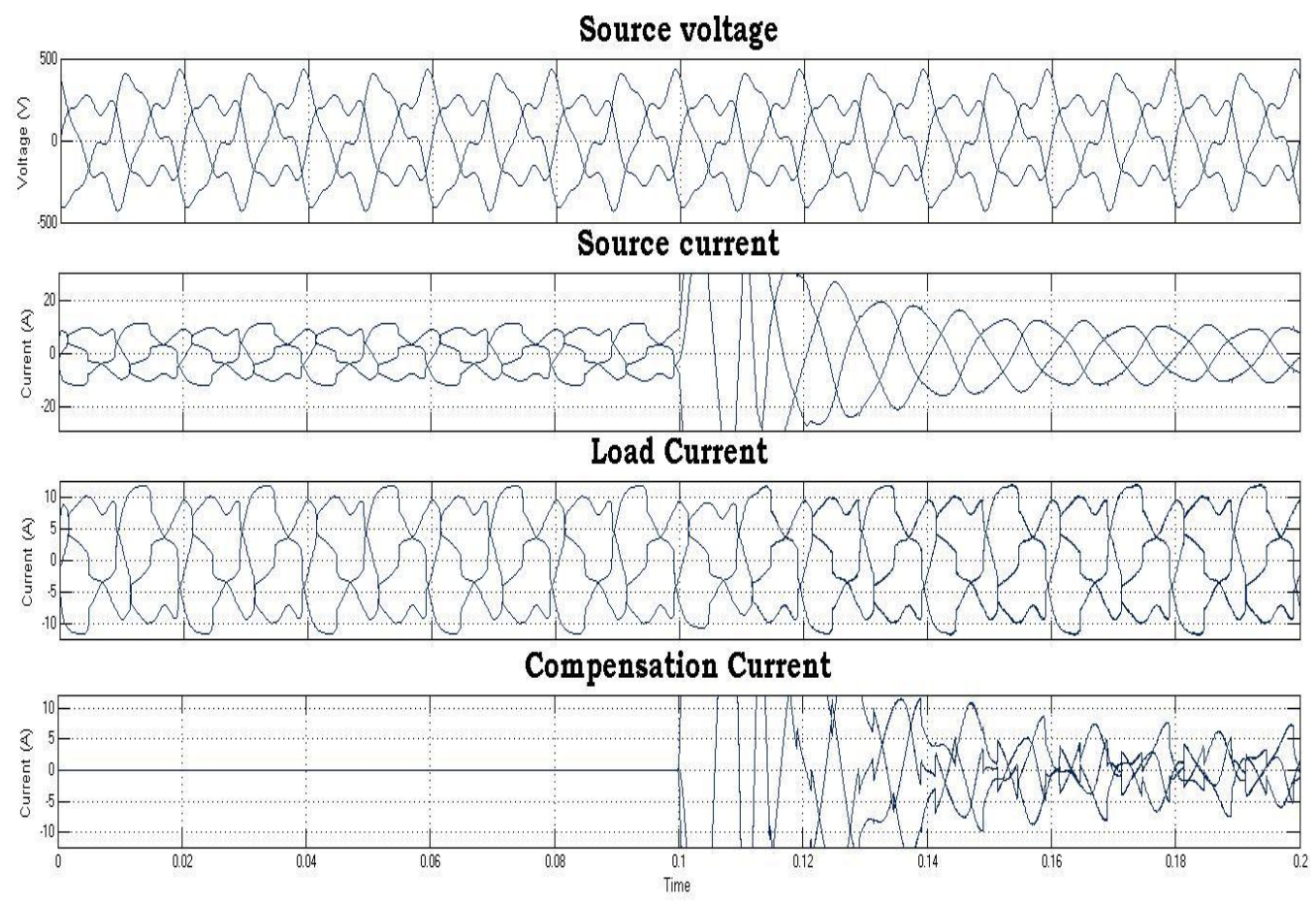

Fig.16. Simulated output wave forms of Source voltage, Source current, Load current and Compensation

Fig.16. shows the output wave form of source voltage and current, load current and compensation current at un-balanced non-sinusoidal source voltage under FUZZY controlled D-Statcom.

Fundamental $(50 \mathrm{~Hz})=10.87, \mathrm{THD}=1.50 \%$

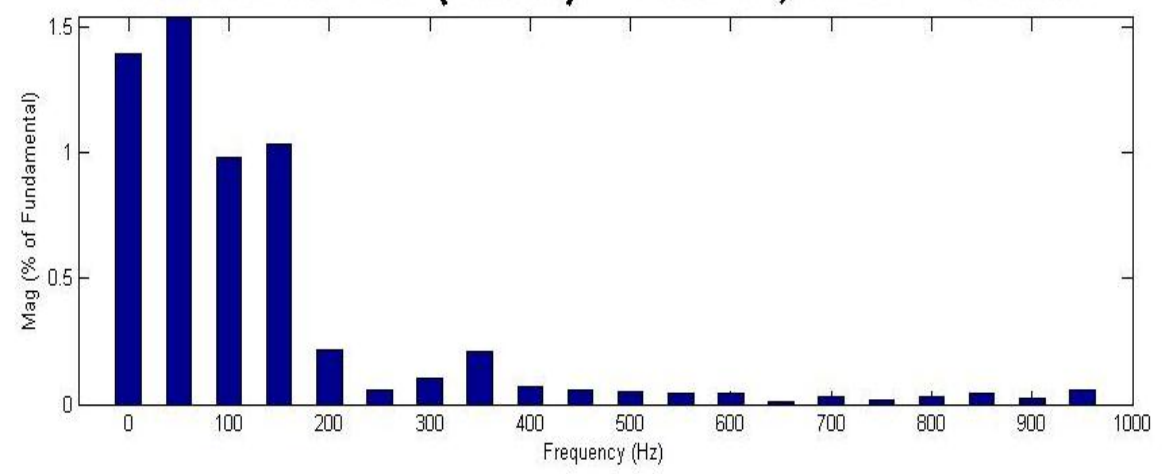

Fig.17. THD of Source Current

Fig.17.Shows the Total Harmonic Distortion of Source Current at Un-Balanced Non-Sinusoidal Source Voltage with Fuzzy Controlled D-Statcom.

\section{CONCLUSION}

In this paper, the renewable sources interconnection with the main supply can influence the power quality at the point of common coupling and can pollute the electrical network with Balanced Sinusoidal and Un-Balanced Non-Sinusoidal voltage distortions and harmonic components which 
exceed the stipulated limits. The proposed fuzzy based D-Statcom is employed to compensate the harmonics and reactive current caused due to the Balanced Sinusoidal and Un-Balanced NonSinusoidal voltage distortions and non-linear load in distribution system. From the simulation analysis the source current harmonics has been reduced to $1.5 \%$ with FUZZY based IPT controlled DSTATCOM but the PI based IPT controlled DSTATCOM only reduces up to $3.41 \%$. Hence it has been proven that fuzzy based IPT controller is suitable for enhancing the power quality at Balanced and Un-Balanced Voltage Variations.

\section{REFERENCES}

[1] A.E. Hammad, Comparing the Voltage source capability of Present and future Var Compensation Techniques in Transmission System, IEEE Trans, on Power Delivery. Volume 1. No.1 Jan 1995.

[2] G.Yalienkaya, M.H.J Bollen, P.A. Crossley, "Characterization of Voltage Sags in Industrial Distribution System", IEEE transactions on industry applications, volume 34, No. 4, July/August, PP.682-688, 1999.

[3] Haque, M.H., "Compensation of Distribution Systems Voltage sags by DVR and D-STATCOM", Power Tech Proceedings, 2001 IEEE Porto, Volume 1, PP.10-13, September 2001.

[4] Anaya-Lara O, Acha E., "Modeling and Analysis Of Custom Power Systems by PSCAD/EMTDC", IEEE Transactions on Power Delivery, Volume 17, Issue: 2002, Pages: 266-272.

[5] Bollen, M.H.J.,"Voltage sags in Three Phase Systems", Power Engineering Review, IEEE, Volume 21, Issue: 9, September 2001, PP: 11-15.

[6] M.Madrigal, E.Acha. "Modeling of Custom Power Equipment Using Harmonics Domain Techniques", IEEE 2000.

[7] R.Meinski, R.Pawelek and I.Wasiak, "Shunt Compensation For Power Quality Improvement Using a D-STATCOM controller Modelling and Simulation”, IEEE Proce, Volume 151, No. 2, March 2004.

[8] J.Nastran , R. Cajhen, M. Seliger, and P.Jereb,"Active Power Filters for Nonlinear AC loads, IEEE Trans.on Power Electronics Volume 9, No.1, PP: 92-96, Jan 2004.

[9] L.A.Moran, J.W. Dixon, and R.Wallace, A Three Phase Active Power Filter with fixed Switching Frequency For Reactive Power and Current Harmonics Compensation, IEEE Trans. On Industrial Electronics. Volume 42, PP:402-8, August 1995. 